\title{
专访催化领域青年学者一一董帆教授
}

《物理化学学报》编辑部

北京大学化学与分子工程学院, 北京 100871

\section{Interview with the Young Scientist of Catalysis: Prof. Fan Dong}

\author{
Editorial Office of Acta Physico-Chimica Sinica \\ College of Chemistry and Molecular Engineering, Peking University, Beijing 100871, China. \\ Email: whxb@pku.edu.cn \\ Published online: November 9, 2020.
}

催化科学与技术对人类经济社会的发展起到了极大的推动作用。调查发现, 大约 $80 \%$ 的化工过程都 需要使用催化剂, 正是因为催化技术的发展, 我们才能够生产足够的化肥、燃料、化工原料以及生活物 资, 保障人类基本需求。催化技术对于经济的直接和间接贡献已高达 $20 \%-30 \%$ 之多, 是人类发展不可 或缺的关键技术。与此同时, 催化学界也不断地在基础和应用方面取得新的突破, 为实现低能耗、低排 放、资源循环利用, 开发新型能源和绿色制品, 解决人类在能源、环境、气候等方面提供了新的选择和 机遇。

本期我们有幸采访了国内催化领域的青年科学家，《物理化学学报》青年编委董帆教授，为我们分 享了他的研究经历及对国内外催化行业的分析。

问题1：请您为读者们简单介绍一下您的科研历程。

回答: 2005年9月我在浙江大学攻读研究生时开始 了我的科研之路，2009-2010年期间在香港理工大 学进行联合培养, 主要从事环境光催化方面的研 究。2010年博士毕业后, 进入重庆工商大学工作至 2018年。2018年下半年调入电子科技大学基础与 前沿研究院, 组建成立了环境与能源催化研究中 心, 学校给我们提供了非常好的平台, 可以围绕兴 趣开展催化研究工作。科研内容包括环境与能源 光催化、光电催化、大气污染控制和催化过程原位 表征技术等。在近十多年的催化研究生涯中, 得到 了包括国家自然科学基金、国家重点研发项目和 国家人才计划的持续资助, 也得到了很多同行的 帮助和支持。

问题2: 是什么把您吸引到催化的研究领域的呢? 在科研的道路上有哪些重要的人影响了您?

回答: 我进入催化研究领域是在攻读研究生阶段 (2005年9月-2010年6月)。浙江大学的导师吴忠标 教授的研究方向是大气污染控制技术, 他给我确
定了光催化净化典型空气污染这个研究方向。从 此, 就开始了催化方面的研究, 非常感谢导师将我 带入到催化研究的大门。在科研道路上, 光催化领 域的多位著名学者对我有较大的影响。在浙大攻 读博士学位期间, 当时通过邮件和电话, 多次联系 了余家国教授。虽然我跟余老师并不相识, 但他总 是热情地帮我解决难题, 在光催化材料合成和机 理认识方面给了我不少指导。另外, 在香港理工大 学访学期间, 合作导师李顺诚教授和香港中文大 学余济美教授, 都给我了很多帮助。李顺诚教授带 着我参加了余济美教授 2010 年1月举办的一个论 坛, 余老师现场给我们演示了光催化的神奇作用, 这让我对光催化研究有了更加浓厚的兴趣。工作 以后, 也经常跟国内外知名学者交流, 包括华中师 范大学张礼知教授, 在科研的方向上给了我建议, 让我在环境光催化方向更加聚焦。总之, 在科研道 路上, 得到了很多催化同行的帮助。

问题3: 中国催化的研究在国际上处于什么位置? 回答: 中国的催化科学与技术始于上世纪初, 经过 
先辈的努力使其经历了发展初期、稳定发展阶段 和快速发展时期。表面科学、纳米科学的出现和高 分辨率表征分析仪器的问世, 大大促进和深化了 催化与不同学科的交叉融合; 同时在不同历史时 期下, 结合国家重大需求, 中国的催化科学与技术 在煤化工、石油冶炼、天然气利用、先进催化材料、 环境保护和清洁能源开发等方面都做出了重大贡 献。经过近三十年的快速发展, 中国在催化领域已 经做出了不少引领性的原创科研成果, 当前已成为 催化大国并正在走向催化强国。

问题 4: 目前催化领域的发展存在的最大的挑战有 哪些？未来有哪些发展趋势?

回答: 目前催化领域面临的最大的挑战包括: (1) 催化本质的探索: 从电子、分子和原子层面, 深入 理解催化反应机理和催化剂表界面作用机理, 包 括热催化、光催化和电催化等不同分支, 这有利于 从原理上指导设计高稳定性和选择性的催化剂; (2)催化技术的应用: 研发低成本高性能的催化组 件、多功能催化剂设计制备及其产业化生产技术, 这可以有效应对能源短缺与环境污染问题。为了 应对催化领域发展的挑战, 未来的发展趋势包括: (1)利用超高分辨分析电镜、原位表征技术、波谱 技术等现代物理化学原位方法和计算化学相结合 的方法去探究催化的本质, 深入理解认识反应机 理, 动态识别催化反应过程活性位点的演变规律; (2)在纳米、原子尺度有效调控催化剂电子结构, 设计并制备高性能催化剂; (3)尽管非均相催化在 工业催化中占主要地位, 但非均相催化、均相催化 和生物催化的融合将成为发展趋势; (4)为了实现 绿色化学和可持续发展, 最终能够真正解决人类 当前面临的难题, 设计绿色经济的催化组件仍是 很重要的一个研究方向。

问题5: 请您列举一些您课题组现阶段主要的研究 方向和学术成果。

回答: 目前我们课题组的研究方向包括: (1)设计 制备绿色、经济、高效的光催化剂, 用于解决环境 污染与清洁能源问题, 具体方向包括光催化降解 挥发性有机污染物(VOCs)、低浓度氮氧化物 $\left(\mathrm{NO}_{x}\right)$ 、光催化 $\mathrm{CO}_{2}$ 还原和光电催化固氮; (2)采用 原位表征技术(例如, 红外光谱和电子自旋共振)揭 示光催化反应过程和机理, 利用密度泛函理论 (DFT)计算模拟催化剂及反应过程中内部电子结 构变化, 通过深入结合实验表征和理论模拟的方 法探究微观结构-内部电子结构-反应机理-反应动
力学-光催化性能之间的关系。目前取得的主要的 学术成果包括: 围绕国家在大气环境保护方面的 重大需求, 针对光催化空气污染物(NO和VOCs)净 化效率不高、反应机理不明确以及中间毒副产物 难控制等关键科学问题, 发展了提升光催化空气 污染物净化效率的新方法, 揭示了光催化净化空 气污染物和 $\mathrm{CO}_{2}$ 还原的反应机理, 提出了调控光催 化过程毒副产物的新策略, 为光催化空气污染物 的安全高效净化奠定了技术基础。取得的科研成 果推动了光催化技术的应用, 并已实现了部分技 术的产业化。

问题6: 您认为催化发展和应用对我们的社会、环 境和经济会有哪些影响?

回答: 催化是化学工业中最重要的技术, 我们衣食 住行中很多原料的生产都离不开催化剂。20世纪, 铁触煤合成氨的发明使人类大量免于饥饿, 欧盟 “地平线 2020 ”计划列出的七大社会挑战中, 有 4 项的应对策略都要用到催化; 在最大宗的 50 种化 工产品中有 30 种的生产需要催化, 而所有化工产 品中这一比例占 $85 \%$ 。正是由于化工生产的迅速发 展, 也带来了一些环境问题, 因此发展高效、绿色 的工业催化剂, 升级当前化工生产技术, 实现清洁 化生产具有重要意义, 这既满足人们生产生活的 需要, 又是不以破坏环境为代价的可持续发展。此 外, 催化技术在环境修复和能源转换方面的迅速 发展, 对当今社会的可持续发展具有重要意义。目 前我国在大气污染物治理方面面临重大挑战, 催 化技术发挥了不可替代的作用。比如, 三效催化、 选择性催化还原(SCR)、催化燃烧等技术, 分别在 汽车尾气、氮氧化物和挥发性有机污染物治理中 起到了核心作用。

问题7: 在您的科研生涯中, 有什么印象深刻的事 情与读者们分享吗?

回答: 有几件事情, 我印象一直都十分深刻, 感觉 我和科学研究之间有一些缘分。这些事情都与实 验研究中的意外发现有关。第一件事情是这样的: 2009年, 浙江大学的导师吴忠标教授派我到香港 理工大学联合培养, 我带着极大的热情去香港做 科研。从 2009 年 9 月 -2010 年 1 月, 整整 4 个月, 几乎 疯狂地做实验, 觉得自己可以大干一场。遗憾的 是, 四个月下来, 合成了至少 100 多个催化剂样品, 但几乎都没有好的催化性能。我甚至开始怀疑自 己的科研能力了。2010年1月底, 我又带着希望开 始测试20多个催化剂样品, 结果还是一样, 我自己 
预期有较好催化性能的样品, 几乎全军覆没! 最后 一个样品, 我记得是实验过程把溶剂乙二醇, 误用 水代替了, 所以觉得是没有用的样品, 因此测试的 时候, 放在最后一个。测完性能后, 发现活性还是 很不错的, 我第一直觉是反应器漏气了。然后开始 排查, 发现并没有漏气。然后开始重复测试, 测了 第二遍、第三遍, 最终确认了催化良好的活性! 这 个时候, 这个意外的结果就引起我的重视了, 实际 上我还不知道这个催化剂是什么。我第二天就用 $\mathrm{XRD}$ 和SEM确认了这个样品的结构, 是玫瑰花状 的碳酸氧铋微球! 微观结构非常漂亮! 这个结果再 次激发了我的热情。接下去就开始对这个新材料 进行系统性的优化, 一直做到离开香港。以这个碳 酸氧铋微球的发现为基础, 我申请到了国家自然 科学基金、省级重点基金等。一直做到现在, 还在 挖掘里面有趣的东西。可以说, 这是我和科研的一 次缘分, 一个错误的实验, 带来了激动人心的结 果, 成为我近十年来最重要的研究方向之一! 在后 来十多年的科研工作中, 类似的事情还发生了几 次, 比如发现 $\mathrm{Bi}$ 金属单质的等离子体光催化现象, 也是源于水浴锅温度设定错误。这说明, 在实验中 仔细记录实验、重视意外实验结果甚至是看似错 误的结果, 是非常重要的。

问题8: 您认为科研人员最重要的品质是什么? 您 对研究生或年轻的科研工作者有什么建议?

回答: 科学研究中没有绝对的真理, 因此我认为科 研人员最重要的品质是敢于质疑, 具备批判性思 维, 这样才能够不断拓展人类知识的边界、推动技 术进步。对于研究生或年轻的科研工作者的建议 有如下几点: (1)做学问要诚实守信, 实事求是,
尊重原始实验数据的真实性; (2)无论从事哪一种 职业, 要想成为本领域中的佼佼者都必须付出比 常人多的时间和精力, 科研工作者更是如此, 要能 够耐得住寂寞, 能够长期坚持不解地在自己感兴 趣的研究方向上努力; (3)培养严密的逻辑思维能 力, 对于科研工作者来说无论是写一篇论文还是 申请基金项目或者重大科研课题, 精准的学术判 断力和严密的逻辑能力就是制胜的法宝。

问题9: 工作之余, 您有什么兴趣爱好?

回答: 在工作之余我喜欢听歌和唱歌。听歌可以放 松身心、消除疲劳, 同时唱歌不仅能使我心情愉 悦, 还是一个增加自我满足感的好方法。此外, 我 还喜欢爬山, 这是一项很好的运动健身活动, 同时 也是一个解压的好方法。

问题 10: 请问您认为什么是期刊发展最重要的因 素?

回答: 我认为学术论文的质量是最重要的因素。优 质的稿源加上严格的审稿可以为读者提供高质量 的学术信息, 这样才能吸引读者, 增加期刊阅读量 及被引频次, 有利于影响因子的增长, 从而也就提 高了期刊的学术影响力; 同时优化编辑出版流程和 期刊流通渠道也很重要, 比如缩短评审流程、提供 高质量审稿意见、在主流学术媒体广泛宣传等, 这 可以为作者通过投稿提高稿件质量提供帮助, 为读 者获取学术前沿信息提供便利; 打造期刊特色、创 建品牌期刊也很重要, 因为品牌是科技期刊核心竞 争力的重要体现, 也是期刊获取国际国内认可并不 断累积学术影响力的最有力武器。

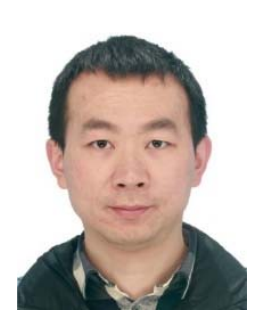

董帆, 1982年8月生, 2010年6月毕业于浙江大学, 香港理工大学访问学者, 从事环 境与能源催化方面的研究。现任电子科技大学基础与前沿研究院教授/博士生导师, 环 境与能源催化研究中心负责人。入选国家青年拔尖人才(2017年), 国家优秀青年科学基 金获得者(2018年), 国务院特殊津贴专家(2019年)。获得教育部自然科学奖一等奖1项、 重庆市自然科学奖二等奖2项、四川省自然科学奖二等奖1项、中国环境科学学会青年科 学家金奖等科技奖励 8 项。获得发明专利授权 20 余项, 出版中英文专著3部。担任Science Bulletin、Chinese Journal of Catalysis、Chinese Chemical Letters、《物理化学学报》、Frontiers in Chemistry 等7本 $\mathrm{SCI}$ 期刊的编委/客座编辑、ACS ES\&T Engineering 的Early Career Board Member和Frontiers in Environmental Chemistry专业主编。主持各类科研项目15项，包括国家自然科学基金项目4项、国家重点 研发计划课题2项、省级重点自然基金1项、省级创新团队项目1项等。在ACS Nano、ACS Catalysis、 Environmental Science \& Technology、Materials Today、Angewandte Chemie、Nano Energy、Science Bulletin、 Applied Catalysis B: Environmental、Journal of Catalysis等期刊上发表SCI论文200余篇, 被SCI引用20000 余次, H index为73。连续3年(2018-2020年)入选科䬥唯安 “全球高被引科学家” 榜单和Elsevier中国高被 引学者。 